\title{
EAS Thermal Neutron Detector Array to Add into LHAASO
}

\author{
Xinhua Ma*, Jlancheng He, Yi Zhang, Youliang Feng, Yiqing Guo, Hongbo Hu, \\ Cheng Liu \\ Key Laboratory of Particle Astrophysics, Institute of High Energy Physics, Chinese Academy of \\ Sciences \\ E-mail: maxheihep.ac.cn
}

Shu-Wang Cui, Bing-Bing Li, Shuai Liu, Cong Shi

The College of Physics Science and Information Engineering, Hebei Normal University

Ye Liu

School of Management Science and Engineering, Heibei University of Economics and Business

Victor Alekseenko, Kirill Levochkin, Vladimir Rulev, Oleg Shchegolev, Yuri Stenkin, Vladimir Stepanov, Ya.V. Yanin

Institute for Nuclear Research, Russian Academy of Science

\section{Chunhui Dong, Rong Zhou}

The College of Physical Science and Technology, Sichuan University

Tian-Lu Chen, Danzengluobu, Qi Gao, Mao-Yuan Liu

Key Laboratory of Cosmic Rays (Tibet University), Ministry of Education

for the LHAASO Collaboration ${ }^{\dagger}$

EN-detectors (Electron-Neutron Detector) can provide detection of both electrons and thermal neutrons generated in ground by secondary hadrons of extensive air shower (EAS). The $400 \mathrm{EN}-$ detector array, so called ENDA (Electron-Neutron Detector Array), was proposed to add into the LHAASO (Large High Altitude Air Shower Observatory) project to improve its capability of EAS hybrid detection. ENDA will be located at the center of the first 1/4 of the KM2A surface scintillator array. Supported by Tibet University, Institute of High Energy Physics of Chinese Academy of Sciences (IHEP CAS) and Institute for Nuclear Research of Russian Academy of Science (INR RAS), 64 EN-detectors of so called ENDA-64 have been produced at Hebei Normal University and pulse shapes are sampled with FADCs made by Sichuan University, networked with the White Rabbit precise clock system. We expect to deploy ENDA-64 (64 EN-detectors) at LHAASO site in 2019. In this paper, we report the results of ENDA-16 (16 EN-detectors) testing run at Yangbajing (YBJ) together with YBJHA offering electrons and muons. It is the first time to obtain the high altitude hybrid detection results between neutrons, electrons and muons in EAS.

36th International Cosmic Ray Conference -ICRC2019-

July 24th - August 1st, 2019

Madison, WI, U.S.A. 


\section{Introduction}

The cosmic ray energy spectrum spans over many decades from about $10^{6} \mathrm{eV}$ to beyond $10^{20}$ $\mathrm{eV}$. It consists of different regions with power law behavior and changes in the power law index. At about 3-5 $\times 10^{15} \mathrm{eV}$, there is a steepening of the spectrum, named "knee", which was measured by several experiments, e.g., KASCADE[四], Tibet AS $\gamma[\mathbb{[}]$ ], ARGO/WFCTA[B]], etc. Although the global features of the all-particle spectrum are reasonably recognized, the spectral shape of each primary component remains an open question related to the interpretation of the experimental data. In particular, high energy hadrons, which constitute EAS (extensive air shower) skeleton, may carry important information for multi-parameter correlation studies, since some hadronic observables, primarily the hadron number/electron number correlation, depend on the nature of the particle inducing EAS [䧃][可][回]. Thus, the detection of high energy hadrons, addressed to improve the discrimination power in these analysis, is highly advisable. Hadrons interacting with ambient matter (air, building, ground, etc.) produce evaporation neutrons due to nuclei disintegration. The neutrons have no charge and lose energy only by scattering. If the medium is a good moderator, i.e., the absorption cross section is much less than the scattering cross section, the neutrons lose energy via scattering down to the thermal ones (moderation process) and then live in the matter until capture. Thermal neutrons are generated abundantly from hadrons on the ground, up to 2 orders of magnitude more than parent hadrons. A way to deal with this problem avoiding the use of huge, expensive HCALs but using thermal neutron detectors, the so-called PRISMA project (PRImary Spectrum Measurement Array), was brought out in [四]. This idea led to the EN-detector developed by Prof. Yu. V. Stenkin at INR RAS for the PRISMA project, made of a mixture of the well-known inorganic scintillator $\mathrm{ZnS}(\mathrm{Ag})$ with ${ }^{6} \mathrm{LiF}$, capable of recording both thermal neutrons and charged particles [ [8] [Q] an EAS array to record simultaneously thermal neutrons and the charged particles in the EAS front [回].

In order to check the performance of the EN-detector at a high altitude site, a small array composed of four EN-detectors (PRISMA-YBJ) was installed inside the hall hosting the ARGO-YBJ experiment [ए]] at the Yangbajing Cosmic Ray Observatory (Tibet, China, $4300 \mathrm{~m}$ a.s.l.). Between ARGO-YBJ and PRISMA-YBJ, the coincident events generated by primary cosmic rays of energies greater than $100 \mathrm{TeV}$ were selected. The EN-detectors were used to record simultaneously thermal neutrons and the EAS electromagnetic component. The density distributions of both components and the total number of thermal neutrons were measured. The correlation of these data with the measurements carried out by ARGO-YBJ confirms the excellent performance of the ENdetector [W]. Besides of research on cosmic ray physics, the EN-detectors can be applied in the other fields concerning neutron flux, such as geophysics. During long stable running, results on the natural thermal neutron flux are obtained. The existence of periodical variations both seasonal and related to the moon month are confirmed for the first time at high altitude. In particular, for synodic moon month the 4th harmonic with amplitude of about $0.5 \%$ and maximum with a delay of about 1 day after syzygies is found [ए2]. It indicates that the EN-detectors can be used to monitor seismic activity. It was identified again by an observation of some anomalies in the dynamics of

\footnotetext{
* Speaker.

${ }^{\dagger}$ for collaboration list see PoS(ICRC2019)1177
} 

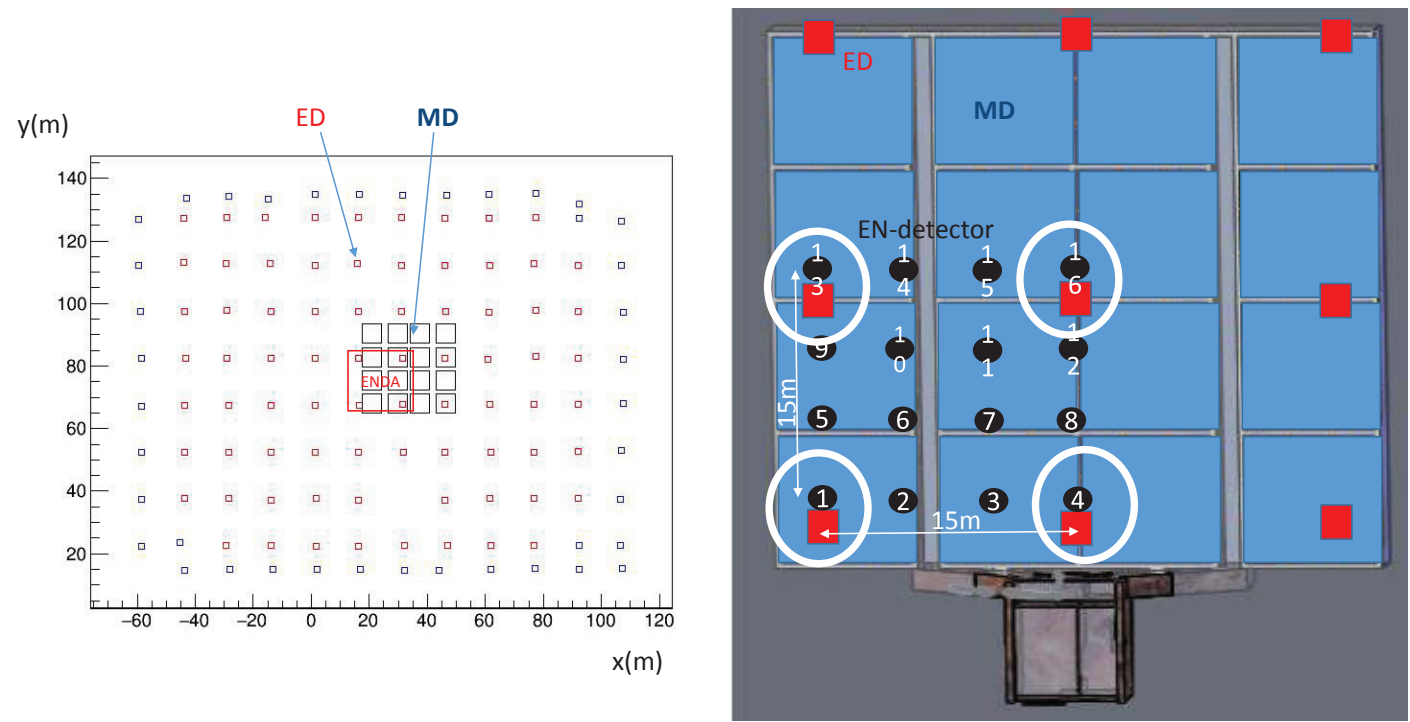

Figure 1: Configuration of ENDA-16 and YBJHA. Left panel: ENDA-16 (the red big square) is at the center of YBJHA-ED and above one quarter of YBJHA-MD. Right panel: the zoom-in drawing of the detectors. Four pairs of EN-detector and EDs (white circles) are at the corners of ENDA-16.

the neutron flux around the time of the catastrophic earthquakes of magnitude $\mathrm{M}=7.8$ happened in Gorkha, Nepal on April 25, 2015, followed by a series of aftershocks of $M>6$ [ए3]]. It shows that the environmental thermal neutron flux is sensitive to changes in tense-deformed crust conditions caused by earthquakes with epicentral distances greater than $500 \mathrm{~km}$.

Because ${ }^{6} \mathrm{Li}$ is raw material of nuclear fission so that its purchase is strong limited by government, we turn to ${ }^{10} \mathrm{~B}$. Although capturing neutron with lower released energy than ${ }^{6} \mathrm{Li},{ }^{10} \mathrm{~B}$ has larger cross section, and ${ }^{10} \mathrm{~B}$ is much easier to be obtained. Moreover, natural Boron contains $19 \%$ versus only $7 \%$ of ${ }^{6} \mathrm{Li}$ in natural Lithium and this allowed us to make a natural boron compound compatible with lithium ones enriched with ${ }^{6} \mathrm{Li}$ up to $90 \%$. A novel type of $\mathrm{ZnS}(\mathrm{Ag}$ ) scintillator alloyed with natural boron compound for thermal neutron capture is developed instead of $\mathrm{ZnS}(\mathrm{Ag})$ with ${ }^{6} \mathrm{LiF}$. Powder of $\mathrm{ZnS}(\mathrm{Ag})$ and $\mathrm{B}_{2} \mathrm{O}_{3}$ alloy is not applied on a sheet of plastic or aluminium as convention, but mixed in liquid silicon rubber which not only easier produced in big size, but also transparent for scintillation lights. In order to check the performance of the new type EN-detectors at a high altitude site, we built a new array of $16 \mathrm{ZnS}\left(\mathrm{Ag}\right.$ ) with $\mathrm{B}_{2} \mathrm{O}_{3}$ EN-detectors (ENDA-16) at Tibet University (TU) in Lhasa, Tibet, China (3700 m a.s.1.) [[4]]. ENDA-16 was moved to YBJ at the end of 2018 to combine with YBJHA for further performance test.

\section{ENDA-16 and YBJHA Experimental Setup}

ENDA-16 consists of $16 \mathrm{EN}$-detectors with distance of $5 \mathrm{~m}$ between adjacent detectors with area of $225 \mathrm{~m}^{2}$. One EN-detectors has sensitive area of $0.35 \mathrm{~m}^{2}$. The peculiar characteristics of the EN-detector output, that are weak and fast signals from charged particles, mainly electrons and 
positrons, compared to high amplitude, slow and delayed signals from thermal neutron capture, make it well suitable for its use in the framework of EAS experiments. The event arrival time is measured by the network timing protocol (NTP) method. NTP is a networking protocol for continuous clock synchronization of computer systems to internet time server. NTP can provide timing with a precision of some $10 \mathrm{~ms}$ to Coordinated Universal Time (UTC). Details of structure and performance of the EN-detector and ENDA-16 were described in [ए4]].

The Yangbajing hybrid array (YBJHA) consists of an electron detector (ED) array [U15] and an muon detector (MD) array [ए6]. The ED array consists of 115 plastic scintillation detectors which are arranged on a square grid with a span of $15 \mathrm{~m}$, covering an area of $20000 \mathrm{~m}^{2}$. Two types of scintillation detectors are used in the ED array: the air light guide detector with area of $0.5 \mathrm{~m}^{2}$ in the middle and wavelength-shift fiber light guide detector with area of $1 \mathrm{~m}^{2}$ as a guard ring. Event trigger timing is provided by a GPS receiver with time precision of $500 \mathrm{~ns}$. the MD array of 900 $\mathrm{m}^{2}$ uses water Cherenkov technique. It consists of 16 MDs with $2.5 \mathrm{~m}$ overburden, and each MD is a waterproof concrete square pool with $7.2 \mathrm{~m}$ in side length and $2.4 \mathrm{~m}$ in height, filled with water $1.9 \mathrm{~m}$ in depth.

The configuration of two arrays is shown in Fig. W. ENDA-16 is at the center of YBJHA$\mathrm{ED}$, above one quarter of the MD array. The four pairs of EN-detectors and EDs at the corners of ENDA-16 are closed to each other so that they can be used to compare measurement of electrons between the two types of detectors.

\section{Results at YBJ}

ENDA-16 and YBJHA were individually triggered by EAS events and coincident events were searched for during off-line data analysis. At the side of ENDA-16, The first level trigger, which is a coincidence of any 2 out of 16 detectors in a time gate of $1 \mu \mathrm{s}$, starts all FADC's. The on-line program analyzes the input data and provides the following second level triggers:

M1, if at least 2 detectors generating the first level trigger measured a signal corresponding to 10 MIPs (minimum ionization particles) or more;

$\mathrm{M} 2$, if the delivered total charge corresponds to more than $125 \mathrm{MIPs}$;

M3, if the total number of recorded neutrons is higher than or equal to 3 .

If any of these conditions is fulfilled thus the pulse shape of all signals is stored in $20 \mathrm{~ms}$ along with the mark of the trigger number. During the off-line analysis, events having $\mathrm{M} 1+\mathrm{M} 2+\mathrm{M} 3$ are selected.

Because timing by the NTP method has not good precision and YBJHA has trigger rate $50 \mathrm{~Hz}$, we have to select high energy events in YBJHA to reduce its rate by using the following selections:

Events can be reconstructed;

$\Sigma \rho_{e}^{E D}>1000$, where $\Sigma \rho_{e}^{E D}$ is sum of electron density of all YBJHA-EDs, which is used to determine primary energy of cosmic ray;

Shower cores drop in square with side of $30 \mathrm{~m}$ at the center of ENDA-16.

Data used in the analysis is from January 1 to May 6, 2019, and actual time of running simultaneously at both sides are 83 days. One example of coincident big events is shown in Fig. ․ The Lateral distribution of Electrons at both arrays are measured very well. several detectors 


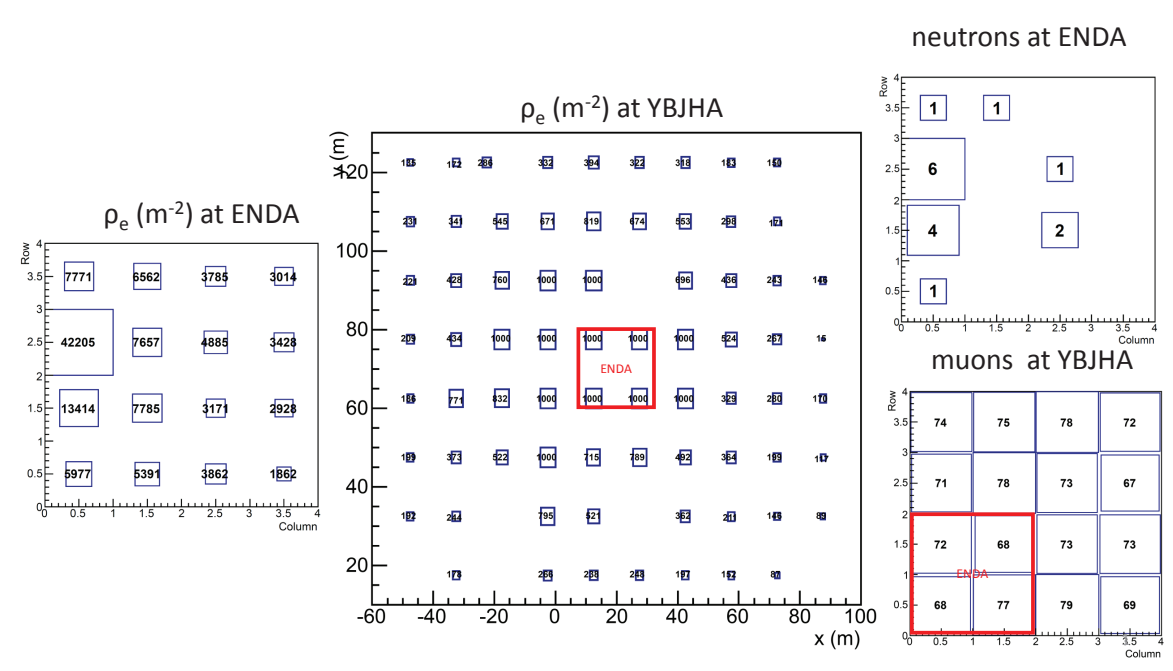

Figure 2: One coincident event between ENDA-16 and YBJHA. Left: distribution of electron density at ENDA $\rho_{e}^{E N D A}$. Middle: distribution of electron density at YBJHA-ED $\rho_{e}^{Y B J H A-E D}$. Right upper: neutron distribution at ENDA. Right lower: muon distribution at YBJHA-MD.

of YBJHA-ED near the shower core reach their maximum measurement capability $\left(\sim 1000 / \mathrm{m}^{2}\right)$. Neutrons is obtained in reasonable lateral distribution. Because the shower core falls into YBJHAMD, the MDs are all saturated.

Correlation of electrons measurement between four pairs of EN-detectors and YBJHA-EDs at the corners of ENDA-16 are obtained (Fig. B]). At $\sim 1000$ electrons $m^{-2}$ YBJHA-EDs are saturated. Linear fitting with function $\lg (\mathrm{y})=\lg (\mathrm{k})+\lg (\mathrm{x})$ is performed and the fitting parameters are listed in Tab. Ш. Moreover, Linear correlation between sum of electron density at ENDA, $\Sigma \rho_{e}^{E N D A}$

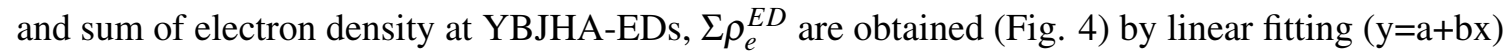
with parameters slope of $0.36 \pm 0.01$ and intersection of $1403 \pm 77$.

\begin{tabular}{|l|l|c|}
\hline Detector of ENDA & Detector of YBJHA-ED & slope $\lg (\mathrm{k})$ \\
\hline 1 & 6 & $-0.020 \pm 0.007$ \\
\hline 4 & 7 & $-0.195 \pm 0.007$ \\
\hline 13 & 2 & $-0.181 \pm 0.006$ \\
\hline 16 & 3 & $0.011 \pm 0.006$ \\
\hline
\end{tabular}

Table 1: Linear fitting parameters of four profiles in Fig. 3.

Correlation between $\Sigma \rho_{e}^{E N D A}$ and neutrons is obtained (Fig. [5). The events are scanned by eye. It is noticed that the events at the right of the straight line are ones with shower cores outside ENDA-16. It means in these events ENDA-16 detects neutrons only far from cores. The cores of the events at the left of the line really fall into ENDA-16 so that more neutrons are recorded. In the same proceeding, cosmic ray composition analysis method to be used in ENDA is described [प]7]. 

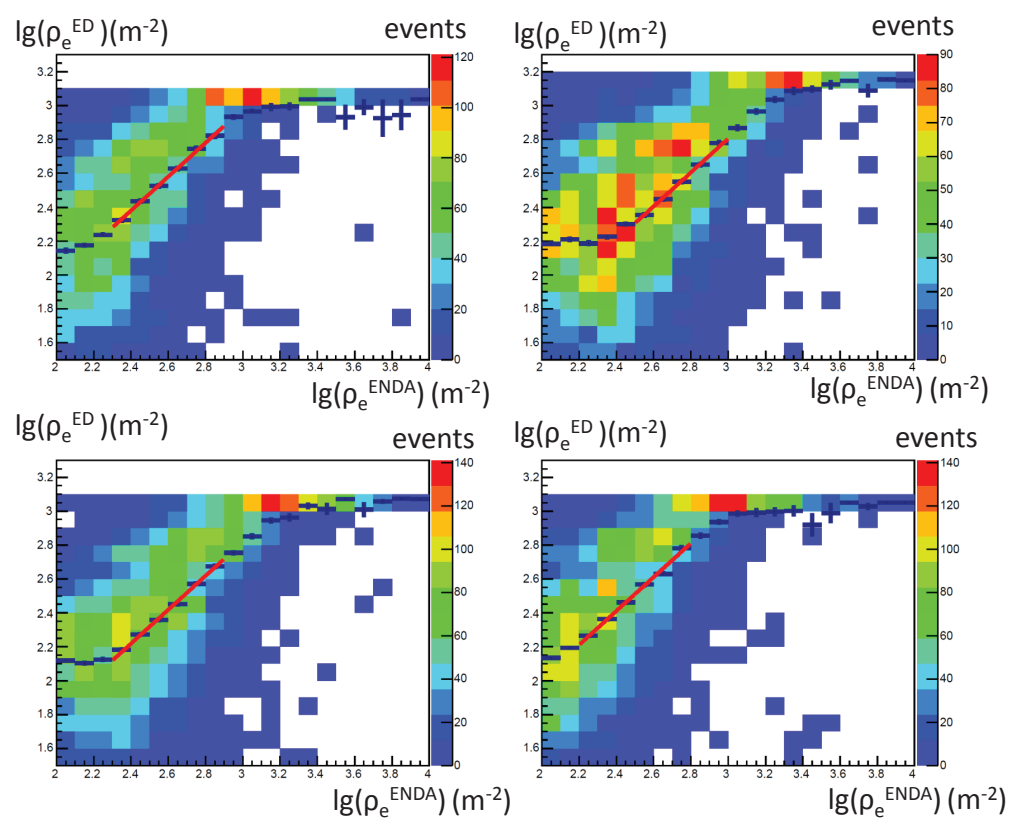

Figure 3: Linear correlation of electrons measurement between four pairs of EN-detectors and YBJHA-EDs. Crosses are profile and straight line is fitting.

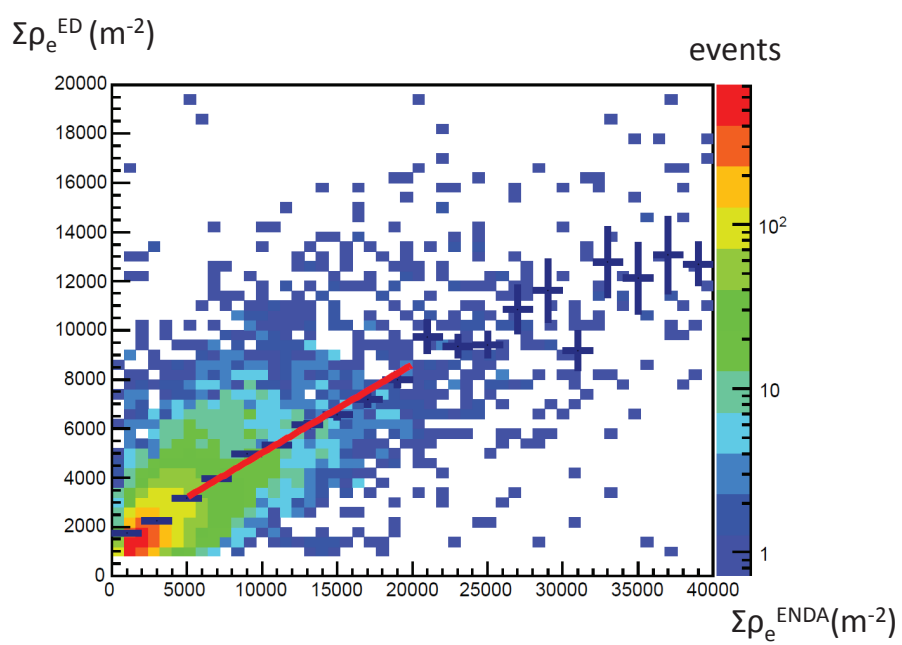

Figure 4: Linear correlation between $\Sigma \rho_{e}^{E N D A}$ and $\Sigma \rho_{e}^{Y B J H A}$. Crosses are profile and straight line is fitting.

\section{Summary and Expectation}

This paper is addressed to test of the new type EN-detector in the framework of EAS measurements at high altitude. The sensitive layer of this detector is basically made of an inorganic $\mathrm{ZnS}(\mathrm{Ag})$ scintillator alloyed with natural Boron compound containing $19 \%$ of the ${ }^{10} \mathrm{~B}$ isotope. The originality of EN-detector lies in simultaneously recording the EAS charged component and the thermal neutrons generated by high energy hadrons. The ENDA-16 array consisting of 16 EN- 


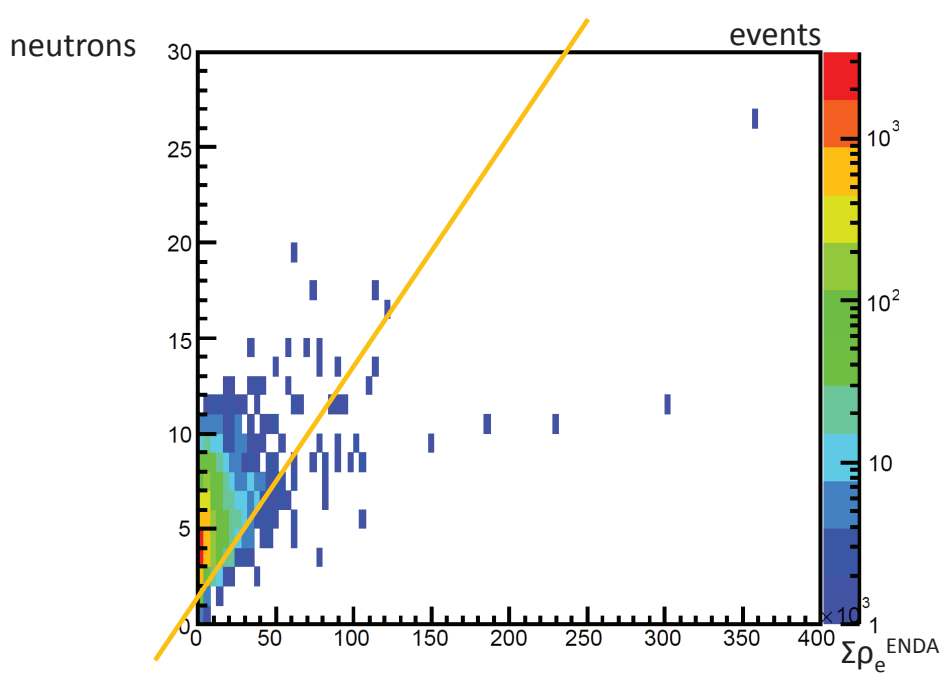

Figure 5: Correlation between $\Sigma \rho_{e}^{E N D A}$ and neutrons. The straight line shows separation between events with cores falling into and out of ENDA-16.

detectors is running smoothly and the first results of coincidence between ENDA-16 and YBJHA confirm that the array works properly at high altitude.

Up to now, ENDA has totally 66 detectors (ENDA-64 and the other two as backup), ready for deploying inside LHAASO in 2019. After achieving good results, ENDA will be extended to 400 detectors with array area of $10000 \mathrm{~m}^{2}$. LHAASO in Haizi Mountain, Daocheng, Sichuan, China (4400 $\mathrm{m}$ a.s.l.) is a large project in deployment to research on high energy gamma ray astrono-

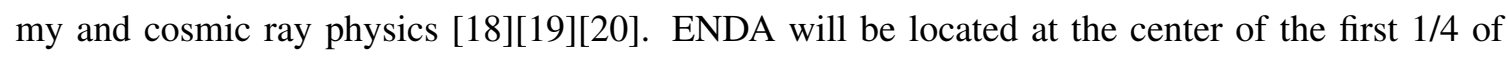
the KM2A surface scintillator array. During the hybrid EAS detection with different parts of the LHAASO experiment, ENDA will provide recording of thermal neutrons from charged hadrons (e.g. $\pi^{+}$and $\pi^{-}$) and electrons near the EAS core, KM2A and WCDA will provide electrons and muons, WCDA++ will provide $\gamma$ family from neutral hadrons ((e.g. $\left.\pi^{0}\right)$ and WFCTA will provide Cerenkov lights. Consequently, LHAASO will perform a detection of full type secondary particles of EAS. Participation of ENDA-400 will enable estimate of hadron content in EAS thus estimating primary particle mass [ [R]], strengthening capability of LHAASO to adequately determine the energy and nature of high energy cosmic rays.

\section{Acknowledgement}

This work was supported in China by the International Partnership Program of Chinese Academy of Sciences (Grant No. 113111KYSB20170055) and by NSFC (No. U1831129, 11873005, 11375052) and in Russia by RFBR (18-02-00339, 16-29-13067_ofi_m), President grant MK1638.2018.2, "The Physics of Fundamental Interactions and Nuclear Technologies" RAS Presidium Program. We also acknowledge the support of Yuan'ai Yu and Hengqiang Zhang in the installation, debugging, and maintenance of the detector. 


\section{References}

[1] T. Antoni, et al. Astroparticle Physics 24 (2005) 1.

[2] M. Amenomori, et al. Physics Letters B 632 (2006) 58.

[3] B. Bartoli, et al. Physical Review D 92 (2015) 092005

[4] Yu.V. Stenkin and J.F. Valdés-Galicia, Modern Physics Letters A 17(26) (2002) 1745.

[5] Yu.V. Stenkin. Nuclear Physics B (Proc. Suppl.) 196 (2009) 293.

[6] Yu.V. Stenkin, et al. Chinese Physics C 37(1) (2013) 015001.

[7] Yu.V. Stenkin and J.F. Valdés-Galicia. Proceedings of 27th International Cosmic Ray Conference, Hamburg (2001) 1453.

[8] Yu.V. Stenkin, D. D. Djappuev, and J. F. Valdés-Galicia. Physics of Atomic Nuclei, 70(6) (2007) 1088.

[9] Yu.V. Stenkin. Nuclear Physics B (Proc. Suppl.), 175-176 (2008) 326.

[10] B. D'Ettorre Piazzoli on behalf of the ARGO-YBJ collaboration, Proceedings of 32nd International Cosmic Ray Conference, Beijing, 12 (2011) 93.

[11] B. Bartoli, et al. Astroparticle Physics 81 (2016) 491́C60.

[12] Y. Stenkin, et al. Pure Appl. Geophys. 174 (2017) 2763íC2771.

[13] Y. Stenkin, et al. Journal of Environmental Radioactivity 208-209 (2019) 105981

[14] B.-B. Li, et al. 2017 JINST 12 P12028

[15] Zhen Wang, et al. Exp Astron (2018) 45:363íc377

[16] Liu, C., et al. Chin. Phys. C 37, 026001 (2013)

[17] X.H. Ma, et al. Cosmic ray mass composition analysis method to be used in the LHAASO-ENDA experiment, the same proceeding.

[18] Z. Cao, Chinese Phys. C 34(2) (2010) 249.

[19] S. Cui, Astroparticle Physics 54 (2014) 86-92.

[20] Y. Liu, The Astrophysical Journal, 826:63 (8pp), 2016.

[21] Yu. V. Stenkin and O. B. Shchegolev, Bulletin of the Russian Academy of Sciences: Physics, 2017, Vol. 81, No. 4, pp. 503íC505. 